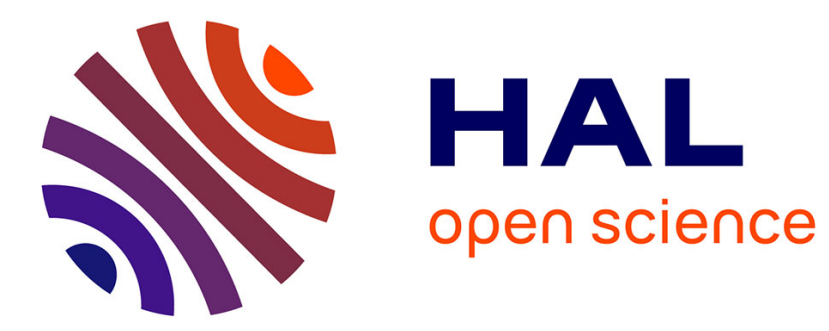

\title{
LMI conditions for topology preservation: applications to multi-agent tasks
}

Irinel-Constantin Morarescu, Mirko Fiacchini

\section{To cite this version:}

Irinel-Constantin Morarescu, Mirko Fiacchini. LMI conditions for topology preservation: applications to multi-agent tasks. Journal of Control Engineering and Technology, 2014, 4 (3), pp.183-191. hal01009619

\section{HAL Id: hal-01009619 \\ https://hal.science/hal-01009619}

Submitted on 19 Jun 2014

HAL is a multi-disciplinary open access archive for the deposit and dissemination of scientific research documents, whether they are published or not. The documents may come from teaching and research institutions in France or abroad, or from public or private research centers.
L'archive ouverte pluridisciplinaire HAL, est destinée au dépôt et à la diffusion de documents scientifiques de niveau recherche, publiés ou non, émanant des établissements d'enseignement et de recherche français ou étrangers, des laboratoires publics ou privés. 


\title{
LMI conditions for topology preservation: applications to multi-agent tasks
}

\author{
Irinel-Constantin Morărescu ${ }^{1}$ and Mirko Fiacchini ${ }^{2}$ \\ ${ }^{1}$ Université de Lorraine, CRAN, UMR 7039 \\ CNRS, CRAN, UMR 7039, Vandoeuvre-lès-Nancy, 54500, France \\ 2 CNRS, GIPSA-lab, UMR 5216, Saint Martin d'Héres, 38402, France, \\ 1 constantin.morarescu@univ-lorraine.fr, ${ }^{2}$ mirko.fiacchini@gipsa-lab.fr
}

\begin{abstract}
In this work we present several implementation strategies answering to different classical problems in multiagent systems. The model under consideration consists of a discrete-time dynamics multi-agent system in which two agents are able to communicate when an algebraic relation between their states is satisfied. As emphasized in the literature, the connectivity of the communication network is essential for global coordination objectives. Thus, the primary goal of our methodology is to characterize the controllers that preserve a given topology allowing the global coordination. In a second step we choose the controller appropriated to the main agreement objective by solving a convex optimization problem associated to the minimization of a well-chosen cost function. Examples concerning full or partial consensus of agents with double integrator dynamics illustrate the implementation of the proposed methodology.
\end{abstract}

Index Terms-Multi-agent systems, LMI, consensus, decentralized control.

\section{INTRODUCTION}

The research on multi-agent systems and decentralized control received an increasing interest during the last decade. This is certainly due to the fact that they found many uses in applications going from biology and medicine to transportation, communication and sociology [Reynolds(2001)], [Blondel et al(2008)], [Colizza(2007)], [Ratmann et al(2009)], [Pavlopoulos et al(2011)]. The consensus problem has been studied under different assumptions such as directed or undirected interaction graph, connections affected or not by delays, discrete or continuous, linear or nonlinear agent dynamics, fixed or dynamic interaction graph, synchronized or desynchronized interactions [Pecora \& Carroll(1998)], [Jadbabaie et al(2003)], [Olfati-Saber \& Murray(2004)], [Ren et al(2005)], [Moreau(2005)], [Olfati-Saber et al(2007], [Morărescu et $\mathrm{al}(2012)]$. It is noteworthy that controlling multi-agent systems in a decentralized manner offers great opportunities for computation and communication cost reduction [Shakkottai \& Srikant(2007)], [Jadbabaie et $\mathrm{al}(2003)$ ], [Ren \& Beard(2005)]. On the other hand the coordination and performances of interconnected systems are related to the network topology. Most of existing works assume the connectivity of the interaction graph in order to guarantee the coordination behavior. However, some works have

The work of I.-C. Morărescu was partially funded by the project ANR13-BS03-0004-02: Computation Aware Control Systems. been oriented towards networks in which the global agreement cannot be reached and only local ones are obtained [Morărescu \& Girard(2011)], [Touri and Nedic(2012)]. Others propose controllers that are able to maintain the network connectivity in order to ensure the global coordination [Zavlanos \& Pappas(2008)], [Bullo et al(2009)], [Fiacchini \& Morărescu(2012)], [Fiacchini \& Morărescu(2014)].

The aim of this paper is to provide implementation strategies for the theoretical tools developed in our previous works [Fiacchini \& Morărescu(2012)], [Fiacchini \& Morărescu(2014)]. Precisely, we consider a multi-agent system with discrete-time dynamics and a dynamic interconnection topology. Two agents are able to communicate if an algebraic relation between their states is satisfied. The connected agents are called neighbors. The agents updates their state in a decentralized manner by taking into account their neighbors state. A connection is preserved as far as the algebraic relation is verified. Thus, we choose a minimal number of interconnections ensuring the network connectivity and making use of set theory [Fiacchini et al(2010)], [Fiacchini et al(2011)], we design a decentralized control law that ensures the satisfaction of the corresponding algebraic constraints.

As shown in [Fiacchini \& Morărescu(2014)], the condition ensuring the topology preservation rewrites as a convex constraint that may be posed in Linear Matrix Inequality (LMI) form, [Boyd et al(1994)], [Boyd \& Vandenberghe(2004)]. Therefore, we not only proposed a new tool for decentralized control but also an easy implementable one. It should be noted that our procedure is quite flexible and, as we shall see, additional global objectives can be addressed. Precisely, we focus on the implementation of the topology preservation, presented in [Fiacchini \& Morărescu(2014)], to tackle specific problems concerning multi-agent systems. The subsystems composing the network are mobile agents moving on the plane and whose communication capability is subject to constraints on their distances. Different coordination tasks, as flocking, consensus and predictive control, are considered and solved employing the LMI conditions for avoiding the connections loss. Numerical illustrative examples allow us to analyze the results and to compare the different control strategies.

The paper is organized as follows. In Section II we formulate the decentralized control problem under analysis. 
Some LMI conditions for network topology preservation are recalled in Section III. Control design strategies for full or partial state consensus of identical systems with doubleintegrator dynamics are discussed in Section IV. In Section V we present some numerical examples illustrating the control strategies proposed in section IV. Some conclusions and remarks on further works are provided at the end of the paper.

Notation: The set of positive integers smaller than or equal to the integer $n \in \mathbb{N}$ is denoted as $\mathbb{N}_{n}$, i.e. $\mathbb{N}_{n}=\{x \in$ $\mathbb{N}: 1 \leq x \leq n\}$. Given the finite set $\mathscr{A} \subseteq \mathbb{N}_{n},|\mathscr{A}|$ is its cardinality. Given a symmetric matrix $P \in \mathbb{R}^{n \times n}$, notation $P>0(P \geq 0)$ means that $P$ is positive (semi-)definite. By $A^{\dagger}$ we denote the left pseudoinverse of the matrix $A$. Given the matrix $T \in \mathbb{R}^{n \times m}$ and $N \in \mathbb{N}, \mathscr{D}_{N}(T) \in \mathbb{R}^{n N \times m N}$ is the blockdiagonal matrix whose $N$ block-diagonal elements are given by $T$, while $\mathscr{D}(A, B, \ldots, Z)$ is the block-diagonal matrix, of adequate dimension, whose block-diagonal elements are the matrices $A, B, \ldots, Z$. Given a set of $N$ matrices $A_{k}$ with $k \in \mathbb{N}_{N}$, denote by $\left\{A_{k}\right\}_{k \in \mathbb{N}_{N}}$ the matrix obtained concatenating $A_{k}$ in column. Given a square matrix $A$, denote with $\lambda_{\text {max }}(A)$ the maximal eigenvalue of $A$.

\section{Problem Statement}

Throughout the paper we consider a multi-agent system with $V \geq 2$ interconnected agents assumed identical. Let us assume that each agent moves in a two dimensional space and is able to select the variation of its velocity. Modelling the input as a velocity variation or, equivalently, the variations along the two Cartesian axis, the dynamics of each $i$-th agent, with $i \in \mathbb{N}_{V}$, along the $x$ axis is given by

$$
\left\{\begin{array}{c}
p_{i}^{x}(k+1)=p_{i}^{x}(k)+t v_{i}^{x}(k), \\
v_{i}^{x}(k+1)=v_{i}^{x}(k)+u_{i}^{x}(k)
\end{array}\right.
$$

where $p_{i}^{x}$ is the position, $v_{i}^{x}$ the velocity, $u_{i}^{x}$ the control input and $t$ the sampling time. So, the overall dynamics of the $i$ th agent along the $x$ axis is given by a linear system with matrices

$$
\bar{A}=\left[\begin{array}{ll}
1 & t \\
0 & 1
\end{array}\right], \quad \bar{B}=\left[\begin{array}{l}
0 \\
1
\end{array}\right]
$$

where the sampling time $t$ has been chosen equal to 0.05 . The dynamics along the $y$ axis are clearly analogous. Then the full dynamics of the $i$-th agent is

$$
x_{i}^{+}=A x_{i}+B u_{i},
$$

with

$$
A=\left[\begin{array}{cc}
\bar{A} & 0 \\
0 & \bar{A}
\end{array}\right], \quad B=\left[\begin{array}{cc}
\bar{B} & 0 \\
0 & \bar{B}
\end{array}\right],
$$

where the state is $x_{i}=\left[p_{i}^{x}(k), v_{i}^{x}(k), p_{i}^{y}(k), v_{i}^{y}(k)\right]^{\top}$ and the input $u_{i}=\left[u_{i}^{x}, u_{i}^{y}\right]^{\top}$.

The usual objectives of the control of multi-agent systems concern the achievement of cooperative tasks by means of decentralized control laws, acting on every agent. In order to pursue such collaborative tasks in a decentralized way, the agents exchange some information. The information available to every agent is supposed to be partial, as only a portion of the overall system is assumed accessible to every agent. We suppose that any agent has access to the state of a neighbor only if a constraint on the distance between them is satisfied. As the loss of the communication network connectivity may hamper the system to reach the global objective, some of such constraints are required to be preserved. Then, the primary problem underlying any cooperative task in the multi-agent context is the connection topology preservation. Theoretical results on this topic, presented in [Fiacchini \& Morărescu(2014)], are recalled hereafter and applied in the following sections.

\section{SET THEORY RESULTS FOR TOPOLOGY PRESERVATION}

In a general framework we can consider the dynamics of the $i$-th agent is given by (2) for all $i \in \mathbb{N}_{V}$, with $A \in \mathbb{R}^{n \times n}$, $B \in \mathbb{R}^{n \times m}$ and where $x_{i} \in \mathbb{R}^{n}$ is the state and $u_{i} \in \mathbb{R}^{m}$ is the control input of the $i$-th agent.

Let us suppose that the initial interconnection topology is given by the graph $G=(\mathscr{V}, \mathscr{E})$ where the vertex set is $\mathscr{V}=\mathbb{N}_{V}$ and the connecting edge set $\mathscr{E} \subseteq \mathscr{V} \times \mathscr{V}$ represents the set of pairs of agents that satisfy a distance-like condition. Precisely, given the real scalar $r>0, d \in \mathbb{N}$ with $d \leq n$ and $T \in \mathbb{R}^{d \times n}$ such that $T T^{\top}$ is invertible, the initial edge set is given by

$$
\mathscr{E}=\left\{(i, j) \in \mathbb{N}_{V} \times \mathbb{N}_{V} \mid\left\|T\left(x_{i}(0)-x_{j}(0)\right)\right\|_{2} \leq r\right\} .
$$

The set of edges that must be preserved is denoted by $\mathscr{N} \subseteq$ $\mathscr{E}$. We suppose that every agent $i$ knows the state of the $j$-th one if and only if $(i, j) \in \mathscr{N}$.

Definition 1: For all $i \in \mathscr{V}$ we define the set of connected neighbors of the $i$-th agent as

$$
\mathscr{N}_{i}=\left\{j \in \mathbb{N}_{V}:(i, j) \in \mathscr{N}\right\} .
$$

Given the set of connections $\mathscr{N}$, the objective is to design a decentralized control law ensuring that none of these connections are lost. In other words, the aim is to design the state-dependent control actions $u_{i}(k)$ independently from $u_{j}(k)$, for all $i, j \in \mathbb{N}_{V}$ and $k \in \mathbb{N}$, such that every connection in $\mathscr{N}$ is maintained.

As usual in multi-agent systems we consider the $i$-th input to be the sum of terms proportional to the distances between agent $i$ and its neighbors. That is, denoting $e_{l, m}=x_{l}-x_{m}$ for all $l, m \in \mathbb{N}_{V}$, we define

$$
u_{i}=\sum_{j \in \mathscr{N}_{i}} K_{i, j}\left(x_{i}-x_{j}\right)=\sum_{j \in \mathscr{N}_{i}} K_{i, j} e_{i, j}
$$

The design of each $u_{i}$ is reduced to the design of the controller gains $K_{i, j}$ chosen such that the link $(i, j)$ is preserved where the dynamics of the $i j$ system results in

$e_{i, j}^{+}=\left(A+B K_{i, j}+B K_{j, i}\right) e_{i, j}+\sum_{k \in \mathscr{N}_{i}}^{k \neq j} B K_{i, k} e_{i, k}-\sum_{k \in \mathscr{N}_{j}}^{k \neq i} B K_{j, k} e_{j, k}$,

for all $i, j \in \mathbb{N}_{V}$. It is not difficult to see that, in the centralized case the dynamics of the error can be imposed by an adequate choice $u_{i}$, for all $i \in \mathbb{N}_{V}$, provided that the agents dynamics is stabilizable. 
The dynamics of the $i j$ system is given by the matrix $A+$ $B K_{i, j}+B K_{j, i}$ if no perturbations due to the presence of other agents are present. Such perturbations, which complicate the decentralized control design, can be bounded within a set depending on the radius $r$ and on the information on the neighbors common to the $i$-th and $j$-th agents.

Consider the sets

$$
\begin{aligned}
& \mathscr{N}_{i, j}=\mathscr{N}_{i} \cap \mathscr{N}_{j}, \\
& \overline{\mathscr{N}}_{i, j}=\mathscr{N}_{i} \backslash\left(\mathscr{N}_{i, j} \cup\{j\}\right), \\
& \overline{\mathscr{N}}_{j, i}=\mathscr{N}_{j} \backslash\left(\mathscr{N}_{i, j} \cup\{i\}\right),
\end{aligned}
$$

then, $\mathscr{N}_{i, j}$ denotes the common neighbors of the $i$-th and the $j$-th agents and $\overline{\mathscr{N}}_{i, j}$ the neighbors of the $i$-th one which are neither $j$ nor one of its neighbors, analogously for $\overline{\mathscr{N}}_{j, i}$. We define the cardinalities

$$
N=2\left|\mathscr{N}_{i, j}\right|+1, \quad \bar{N}=\left|\overline{\mathscr{N}}_{i, j}\right|+\left|\overline{\mathscr{N}}_{i, j}\right|,
$$

where the indices are avoided here and in the following definitions to improve the readability.

The problem addressed in this paper can be state as follows.

Problem 1: Design a procedure to find at each step a condition on the decentralized control gains $K_{i, j}$, with $i, j \in$ $\mathbb{N}_{V}$ such that the following algebraic relation is satisfied

$$
\left\|T e_{i, j}^{+}\right\|_{2}<r, \quad \forall(i, j) \in \mathscr{N},
$$

if the constraints

$$
\begin{array}{ll}
\left\|T e_{i, k}\right\|_{2} \leq r, & \forall k \in \overline{\mathscr{N}}_{i, j}, \\
\left\|T e_{j, k}\right\|_{2} \leq r, & \forall k \in \overline{\mathscr{N}}_{j, i},
\end{array}
$$

hold.

In order to ease the presentation, we introduce different notations for the controller gains.

Definition 2: Denote with $\mathbf{E}_{i, j} \in \mathbb{R}^{n N}$ the vector obtained concatenating $e_{i, j}$ with all $e_{i, k}$ and $e_{j, k}$ where $k \in \mathscr{N}_{i, j}$. Denote with $\check{\mathbf{K}}_{i, j} \in \mathbb{R}^{m \times n(N-1)}$ the matrix obtained concatenating $K_{i, k}$ and $-K_{j, k}$ where $k \in \mathscr{N}_{i, j}$ and with $\hat{\mathbf{K}}_{i, j} \in \mathbb{R}^{m \times n \bar{N}}$ the vector obtained concatenating all $K_{i, k}$ where $k \in \overline{\mathscr{N}}_{i, j}$ and $-K_{j, k}$ where $k \in \overline{\mathscr{N}}_{j, i}$. We also define

$$
\begin{aligned}
& \Delta=T\left[A+B \check{K}_{i, j}, B \check{\mathbf{K}}_{i, j}\right] \mathscr{D}_{N}(T)^{\dagger} \in \mathbb{R}^{d \times d N} \\
& \Gamma=T B \hat{\mathbf{K}}_{i, j} \mathscr{D}_{\bar{N}}(T)^{\dagger} \in \mathbb{R}^{d \times d \bar{N}} \\
& Z=\mathscr{D}_{N}(T) \mathbf{E}_{i, j} \in \mathbb{R}^{d N}
\end{aligned}
$$

where $\check{K}_{i, j}=K_{i, j}+K_{j, i}$.

We recall here an important contribution presented in [Fiacchini \& Morărescu(2014)], namely the sufficient condition for the constraint (6) to hold.

Theorem 1: Problem 1 admits solutions if there exists $\Lambda=$ $\mathscr{D}\left(\lambda_{1} I_{d}, \ldots, \lambda_{\bar{N}} I_{d}\right)$ with $\lambda_{k} \geq 0$, for all $k \in \mathbb{N}_{\bar{N}}$ such that

$$
\left[\begin{array}{ccc}
r^{2}-r^{2} \sum_{k \in \mathbb{N}_{\bar{N}}} \lambda_{k} & 0 & Z^{\top} \Delta^{\top} \\
0 & \Lambda & \Gamma^{\top} \\
\Delta Z & \Gamma & I_{d}
\end{array}\right]>0 .
$$

Furthermore, any solution $(\Delta, \Gamma)$ of the previous LMI defines admissible controller gains for the Problem 1 .

The quantity $\delta=\sum_{k \in \mathbb{N}_{\bar{N}}} \lambda_{k}$ can be geometrically interpreted as a bound on the perturbation generated in the $i j$ dynamics by the non-common neighbors. Precisely, the effect of the non-common neighbors can be modelled as a perturbation on the $i j$ system bounded by an ellipsoid determined by $T^{\top} T$ and of radius $\sqrt{\delta} r$. Therefore the condition $\delta<1$, implicitly imposed by (9), is necessary to ensure the preservation of the connection $(i, j)$.

\section{Applications to DeCENTRALIZED CONTROL OF MULTI-AGENT SYSTEMS}

In this section we illustrate the application of our results, published in [Fiacchini \& Morărescu(2014)] and recalled in Section III, for controlling the multi-agent system presented in Section II. Different strategies (based on optimal and predictive control) to achieve the collaborative objectives are presented hereafter and numerically implemented.

Denote $p_{i, j}^{x}=p_{i}^{x}-p_{j}^{x}, v_{i, j}^{x}=v_{i}^{x}-v_{j}^{x}, p_{i, j}^{y}=p_{i}^{y}-p_{j}^{y}, v_{i, j}^{y}=$ $v_{i}^{x}-v_{j}^{x}$ and

$$
e_{i, j}=\left[p_{i, j}^{x}, v_{i, j}^{x}, p_{i, j}^{y}, v_{i, j}^{y}\right]^{\top}=x_{i}^{\top}-x_{j}^{\top},
$$

and $u_{i, j}=\left[u_{i}^{x}-u_{j}^{x}, u_{i}^{y}-u_{j}^{y}\right]^{\top}$. The control inputs are given by (3) and Definition 2 with feedback gains

$$
\check{K}_{i, j}=\left[\begin{array}{cccc}
k_{i, j}^{p^{x}} & k_{i, j}^{v^{x}} & 0 & 0 \\
0 & 0 & k_{i, j}^{p^{y}} & k_{i, j}^{v^{y}}
\end{array}\right],
$$

for all $(i, j) \in \mathscr{N}$. Once obtained a value for $\check{K}_{i, j}$, we define the nominal selection $K_{i, j}=K_{j, i}=0.5 \check{K}_{i, j}$ for all $(i, j) \in \mathscr{N}$.

Moreover, the following constraint on the norm of $\check{K}_{i, j}$ is imposed

$$
\check{K}_{i, j}^{\top} \check{K}_{i, j} \leq I_{n},
$$

to limit the effect of the control of the $i j$ nominal system on the neighbors. Recall, in fact, that the perturbation on the neighbors of the agents $i$ and $j$ depends on their states and on the gains $K_{i, j}$ and $K_{j, i}$.

Remark 1: It is worth recalling that the $i j$ system, with $(i, j) \in \mathscr{N}$, considered and analysed hereafter, is the generic model of connected agents. The overall system concerns several analogous models, one for every pair of connected agents, represented by the elements of $\mathscr{N}$. Hence, several distance constraints have to be maintained and several local optimization problems to be solved.

\section{A. Topology preservation constraint}

We suppose that the distance between two agents must be smaller than or equal to $r$ to allow them to communicate. Thus the topology preservation problem consists of upperbounding by $r$ the euclidean distance between the connected neighbors. The constraint on the state of the $i j$ system to preserve is

$$
p_{i, j}^{x}(k)^{2}+p_{i, j}^{y}(k)^{2} \leq r^{2}
$$

Notice that the effect of the inputs $u_{i}^{x}$ and $u_{i}^{y}$ at time $k$ has no influence on $p_{i}^{x}$ and $p_{i}^{y}$ at time $k+1$ (see (1)). Thus, any algebraic condition involving the positions $p_{i}^{x}, p_{i}^{y}$ of the systems at $k+1$ would not depend on the control action $u_{i}^{x}, u_{i}^{y}$ at time $k$. From the computational point of view, every constraint concerning only the agents positions, would lead 
to LMI conditions independent on the variable $\check{K}_{i, j}$. Then the results provided in Theorem 1 are not applicable directly in this case for the state at time $k+1$. On the other hand, the controls $u_{i}^{x}(k), u_{i}^{y}(k)$ affect the position (and the velocity) at time $k+2$ and a condition on the feedback gain $\check{K}_{i, j}$ to ensure the preservation of the $(i, j)$ connection at time $k+2$ can be posed. The distance constraint can be imposed on the states at $k+2$, as nothing can be done at time $k$ in order to prevent its violation at time $k+1$. Then a constraint on $e_{i, j}(k)$ can be determined characterizing the region of the state space such that $p_{i, j}^{x}(k)^{2}+p_{i, j}^{y}(k)^{2} \leq r^{2}$ and $p_{i, j}^{x}(k+1)^{2}+p_{i, j}^{y}(k+1)^{2} \leq r^{2}$ in terms of matrix $T$. Since the former constraint does not involve the input, only $p_{i, j}^{x}(k+1)^{2}+p_{i, j}^{y}(k+1)^{2} \leq r^{2}$ might be taken into account for the control design.

Proposition 1: The condition (13) holds at time $k+2$ if and only if we have that $\left\|T e_{i, j}(k+1)\right\|_{2} \leq r$ with

$$
T=\left[\begin{array}{llll}
1 & t & 0 & 0 \\
0 & 0 & 1 & t
\end{array}\right] .
$$

Proof: The region of the space of $e_{i, j}(k)$ such that the topology constraint (13) is satisfied at $k+1$ is given by $p_{i, j}^{x}(k+1)^{2}+p_{i, j}^{y}(k+1)^{2} \leq r^{2}$, which is equivalent to $\left\|T e_{i, j}(k)\right\|_{2} \leq r$ for $T$ as in (14). Hence imposing that the system error state belongs to such a region at $k+1$ implies assuring that the distance between the agents $i$-th and $j$-th is smaller than or equal to $r$ at $k+2$, preserving the topology at $k+2$. Then $p_{i, j}^{x}(k+2)^{2}+p_{i, j}^{y}(k+2)^{2} \leq r^{2}$ if and only if

$$
\left\|T e_{i, j}(k+1)\right\|_{2}=\left\|T\left(A e_{i, j}(k)+B u_{i, j}(k)\right)\right\|_{2} \leq r,
$$

with $T$ as in (14).

Proposition 1, then, implies that the topology preservation constraint for time $k+2$ can be expressed in terms of $e_{i, j}(k)$ and the input $u_{i, j}(k)$. The results presented in Theorem 1 , with $T$ as in (14), allow to characterize the sets of feedback gains ensuring the satisfaction of the distance constraint at $k+2$, for every pair of connected neighbors $i$ and $j$. Such set would depend on the current state $e_{i, j}(k)$ and on the gains designed to compensate the errors and enforce the topology preservation.

\section{B. Relevant multi-agents applications}

Among the local feedback gains which guarantee the connection preservation, different selection criteria can be applied, depending on the collaborative task to be achieved. Hereafter three popular criteria are illustrated and analysed.

1) Full state consensus: The first criterion is to select the feedback gain, among those satisfying (9), to achieve the full state agreement. In other words, the objective in this case is to both steer all the agents at the same point and align all the velocities without loosing any connection. One possibility is to compute at any sampling instant the matrix $\breve{K}_{i, j}$ minimizing a sum of nominal values of the position distance at $k+2$ and of the speed difference at $k+1$. By nominal values we mean the values of positions and speeds in absence of the perturbation on the $i j$ system due to the other agents. Then, given the positive weighting parameters $q_{p}, q_{v} \in \mathbb{R}$, the cost to minimize is

$$
\begin{aligned}
& Q_{c}\left(e_{i, j}(k), \check{K}_{i, j}\right)=q_{p}\left(p_{i, j}^{x}(k+2)^{2}+p_{i, j}^{y}(k+2)^{2}\right)+ \\
& \quad+q_{v}\left(v_{i, j}^{x}(k+1)^{2}+v_{i, j}^{y}(k+1)^{2}\right) .
\end{aligned}
$$

Proposition 2: Any optimal solution of the convex optimization problem

$$
\begin{aligned}
& \min _{\Gamma, \Lambda, \breve{K}_{i, j}, M} e_{i, j}(k)^{\top} M^{\top} M e_{i, j}(k) \\
& \text { s.t. } \quad(8),(9),(11) \text {, } \\
& {\left[\begin{array}{cc}
I_{n} & \check{K}_{i, j}^{\top} \\
\check{K}_{i, j} & I_{m}
\end{array}\right] \geq 0}
\end{aligned}
$$

with

$$
M=\left[\begin{array}{cccc}
q_{p} & q_{p} t & 0 & 0 \\
0 & q_{v} & 0 & 0 \\
0 & 0 & q_{p} & q_{p} t \\
0 & 0 & 0 & q_{v}
\end{array}\right]\left(A+B \check{K}_{i, j}\right),
$$

and $T$ as in (14), minimizes the cost (15) subject to the norm gain constraint (12) and the distance constraints (13) at $k+2$.

Proof: From standard algebraic manipulation, it can be proved that $Q_{c}\left(e_{i, j}(k), \check{K}_{i, j}\right)=e_{i, j}(k) M^{\top} M e_{i, j}(k)$. From (8), (9), and Proposition 1, any feasible solution of (16) assures the distance constraints to hold at $k+2$. Finally, (12) is equivalent to the last LMI constraint in (16).

2) Partial state consensus: flocking: An alternative objective, often considered in the framework of decentralized control, is to steer a part of the state $e_{i, j}$ to zero, for all $(i, j) \in \mathscr{N}$. In particular, the problem of flocking consists in designing a decentralized control such that the difference between the speeds of every pair of connected agents converges to zero, avoiding violations of the distance constraints. Then, if the graph $G=(\mathscr{V}, \mathscr{N})$ is preserved connected and the speed differences converge to zero, the agents reach and maintain the flocking. For this purpose, the cost to minimize is a measure of the difference between neighbors speeds, for instance

$$
Q_{f}\left(e_{i, j}(k), \check{K}_{i, j}\right)=v_{i, j}^{x}(k+1)^{2}+v_{i, j}^{y}(k+1)^{2} .
$$

This is achieved by solving a convex optimization problem analogous to (16), as stated in the proposition below. The proof is avoided since similar to the one of Proposition 2.

Proposition 3: Any optimal solution of the convex optimization problem (16) with

$$
M=\left[\begin{array}{llll}
0 & 1 & 0 & 0 \\
0 & 0 & 0 & 1
\end{array}\right]\left(A+B \check{K}_{i, j}\right),
$$

and $T$ as in (14), minimizes the cost (18) subject to the norm gain constraint (12) and the distance constraints (13) at $k+2$.

Clearly, changing opportunely the matrix $M$ would permit to regulate different part of the state of the $i j$ system and also any linear combination of the state.

3) Predictive control: Finally, we present another interesting optimization criterion. One of the most popular control technique suitable for dealing with control in presence of hard constraints is the predictive control. These control strategies exploit the prediction of the system evolution and the receding horizon strategy to react in advance in order to prevent the constraint violations and to avoid the potentially 
dangerous regions of the state space. Moreover, the control input that would generate the optimal trajectory, among the admissible ones, is usually computed and applied. In general, the longer is the prediction horizon, the higher is the capability of preventing unsafe regions and constraint violations. Based on this idea, we propose to optimize a measure of the future state position, in order to react in advance and prevent the states to approach the limits of the distance constraints. In particular we minimize a measure of the nominal distance between the positions of the $i$ th and $j$-th agents at time $k+3$ in function of the input gain at time $k$, that is $\left(p_{i, j}^{x}(k+2)+t v_{i, j}^{x}(k+1)\right)^{2}+\left(p_{i, j}^{y}(k+\right.$ $\left.2)+t v_{i, j}^{y}(k+1)\right)^{2}$. The control horizon can be extended to values higher than 3 , but the predicted state $e_{i, j}(k+N)$ would depend on the future inputs and the cost would result in a non-convex function of $\check{K}_{i, j}$. A simplifying hypothesis can be posed to obtain a suboptimal control strategy but with greater prediction capability. Let us denote the horizon $N_{p} \in \mathbb{N}$ and suppose that only the nominal control action $u_{i, j}(k)=\check{K}_{i, j} e_{i, j}(k)$ is applied, i.e. $u_{i, j}(k+p)=0$ for $p \in \mathbb{N}_{N_{p}}$. The minimization of the nominal position at $k+N_{p}$, i.e.

$$
Q_{p}\left(e_{i, j}(k), \check{K}_{i, j}\right)=p_{i, j}^{x}\left(k+N_{p}\right)^{2}+p_{i, j}^{y}\left(k+N_{p}\right)^{2},
$$

leads to a suboptimal control with high predictive power.

Proposition 4: Any optimal solution of the convex optimization problem (16) with

$$
M=T+\left(N_{p}-1\right) t\left(\left[\begin{array}{cccc}
0 & 1 & 0 & 0 \\
0 & 0 & 0 & 1
\end{array}\right]+\check{K}_{i, j}\right),
$$

and $T$ as in (14), minimizes the cost (20) subject to the norm gain constraint (12) and the distance constraints (13) at $k+2$.

The benefits of the prediction-based strategy will be highlighted in the numerical examples section.

\section{NumERICAL EXAMPLES}

Two numerical case of studies and different global objectives are considered in this illustrative section. The simulations have been performed in MATLAB. At every time instant, the solution of the convex problem, based on the LMI conditions for connection preservation, is solved independently for each agent. Every connection $(i, j)$ is considered by the agents $i$ and $j$ leading to the same LMI-constraint. The only information employed by any agent is, as assumed above, the knowledge of the states of the neighbors involved in the connections to be preserved. Every agent computes and applies a feedback control according to the LMI conditions, no information interchange between agents is considered, although it could and should in future works.

Example 1: Firstly, let us consider the problem of flocking for a simple system consisting of three interconnected agents. Suppose that the 2-nd agent is a common neighbor of both the 1-st and the 3-rd one, which are not neighbors each other. The distance bound assuring the connections between the agents is $r=2$. The initial states are

$$
\begin{aligned}
& x_{1}(0)=\left[\begin{array}{llll}
2.43 & 1.215 & 0 & 0
\end{array}\right]^{\top}, \\
& x_{2}(0)=\left[\begin{array}{llll}
1.215 & 0 & -1.215 & -1.8225
\end{array}\right]^{\top}, \\
& x_{3}(0)=\left[\begin{array}{llll}
0 & -0.486 & 0.243 & 0
\end{array}\right]^{\top},
\end{aligned}
$$

and then

$$
\begin{aligned}
& e_{1,2}=\left[\begin{array}{llll}
1.215 & 1.215 & 1.215 & 1.8125
\end{array}\right]^{\top}, \\
& e_{3,2}=\left[\begin{array}{llll}
-1.215 & -0.486 & 1.458 & 1.8225
\end{array}\right]^{\top} .
\end{aligned}
$$

The distances between the connected agents, the 12 system and the 23 one, are close to the boundary as seen in Figure 1. The initial speeds are pushing the agents away one of each others, towards the boundary of the connection region. Although the 2-nd and the 3-rd agent are very close to the constraint limit, the control succeeds to reduce their relative speed and to stop their drift within just few sampling times. The same happens with the speed difference between the 1st and 2-nd agent. Therefore, the connection graph is kept and one gets a formation that moves with the same speed with relative position distances close to the connection limit, see Figure 2. Obviously, increasing the initial distances between neighbors or the initial velocities the control problem formulated in Section IV-B.2 may not have solutions and the graph connectivity is lost.
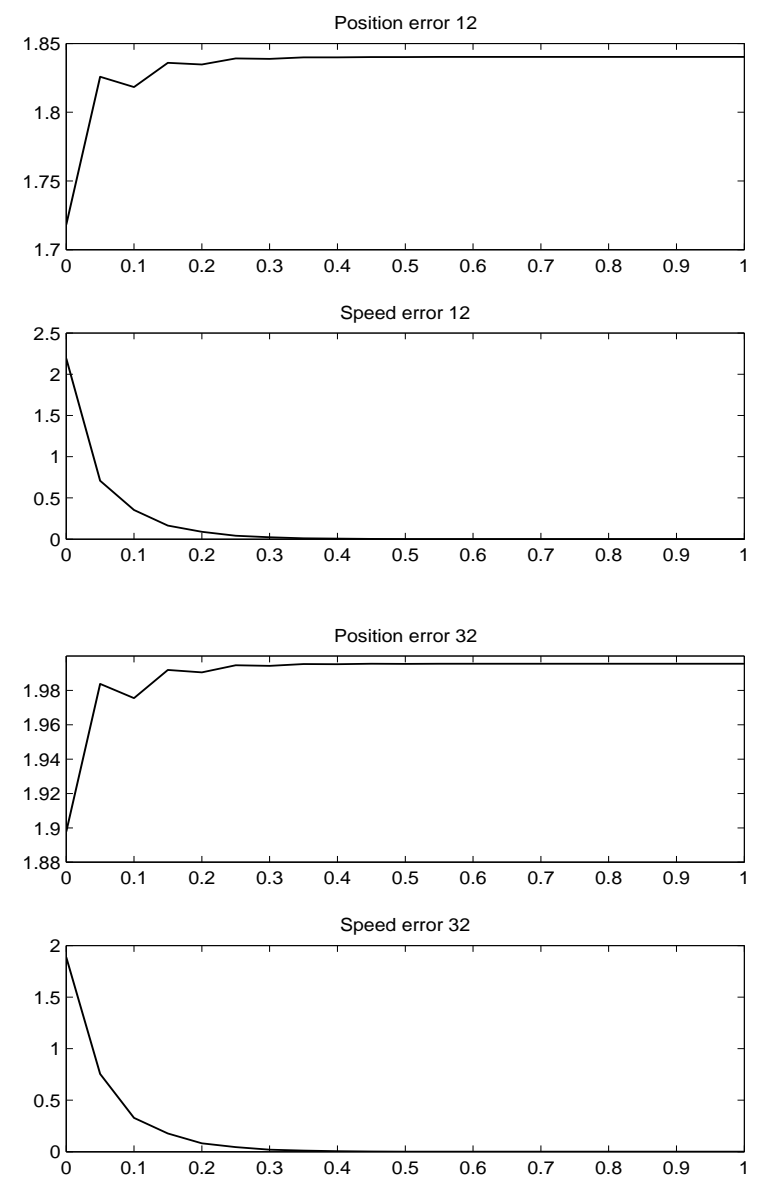

Fig. 1. Flocking: errors 12 and 32.

Example 2: Consider now the six interconnected agents with the initial conditions given in [Martin \& Girard(2010)] and connected by the minimal robust graph computed in the same work. That is: $\mathscr{N}=\{(1,2),(2,3),(3,4),(4,5),(5,6)\}$, $r=3.2$ and initial conditions: 


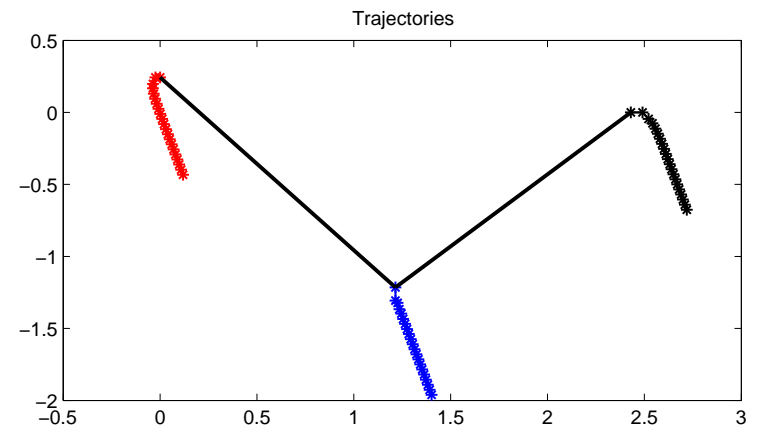

Fig. 2. Flocking: trajectories.

$$
\begin{array}{lll}
x_{1}(0)=\left[\begin{array}{llll}
-4 & -v_{0} & 3 & 0
\end{array}\right]^{\top}, & x_{6}(0)=\left[\begin{array}{llll}
4 & v_{0} & 3 & 0
\end{array}\right]^{\top}, \\
x_{2}(0)=\left[\begin{array}{llll}
-2 & -v_{0} & 2 & 0
\end{array}\right]^{\top}, & x_{5}(0)=\left[\begin{array}{llll}
2 & v_{0} & 2 & 0
\end{array}\right]^{\top}, \\
x_{3}(0)=\left[\begin{array}{llll}
-1 & -v_{0} & 0 & 0
\end{array}\right]^{\top}, & x_{4}(0)=\left[\begin{array}{llll}
1 & v_{0} & 0 & 0
\end{array}\right]^{\top},
\end{array}
$$

where $v_{0}$ is used as a parameter to analyze the maximal initial speed that may be dealt with by different control strategy. It is noteworthy that, as shown in [Martin \& Girard(2010)], for the classical consensus algorithm the preservation of the minimal robust graph is guaranteed for a critical speed value $v_{c} \simeq 0.23$. Nevertheless, it is numerically shown that the sufficient condition is conservative since for $v_{0}=1.5 v_{c}$ (generating approximately a 4 times higher global velocity disagreement) the robust graph is not broken. We also note that the classical consensus algorithm is not able to preserve the connectivity when the global disagreement is 5 times superior to the one guaranteeing the consensus (i.e. $\left.v_{0}>2.1 v_{c}\right)$.

In the sequel, we show that our design allows to increase considerably the initial speed value (and consequently the initial global disagreement) avoiding the loss of connections. Let us first give the initial error vectors between the states of the neighbors:

$$
\begin{gathered}
e_{1,2}(0)=\left[\begin{array}{llll}
-2 & 0 & 1 & 0
\end{array}\right]^{\top}, \quad e_{5,6}(0)=\left[\begin{array}{llll}
-2 & 0 & -1 & 0
\end{array}\right]^{\top}, \\
e_{2,3}(0)=\left[\begin{array}{llll}
-1 & 0 & 2 & 0
\end{array}\right]^{\top}, \quad e_{4,5}(0)=\left[\begin{array}{llll}
-1 & 0 & -2 & 0
\end{array}\right]^{\top}, \\
e_{3,4}(0)=\left[\begin{array}{llll}
-2 & -2 v_{0} & 0 & 0
\end{array}\right]^{\top} .
\end{gathered}
$$

\section{A. Flocking}

The control problem formulated in Section IV-B.2 has admissible solutions for $v_{0}=19 v_{c}$ and the connection between the third and the fourth agent is lost for $v_{0}=20 v_{c}$ as shown in Figure 4. It is worth noting that the control acts like springs between agents' velocities (compare the bottom of Figures 3, 4 and 5). First, the control cancels the speed difference between neighbors with opposite velocities creating a speed disagreement in both symmetric branches of the graph. Next, it cancel the disagreement between 2-nd and the 3-rd agent and between the 4-th and 5-th one, mimicking a gossiping procedure where the choice of active communication link is given by the error between neighbors speeds. Doing so, either the flocking is reached before the connectivity is lost, or the graph splits into two groups that will independently agree to two different velocity values.
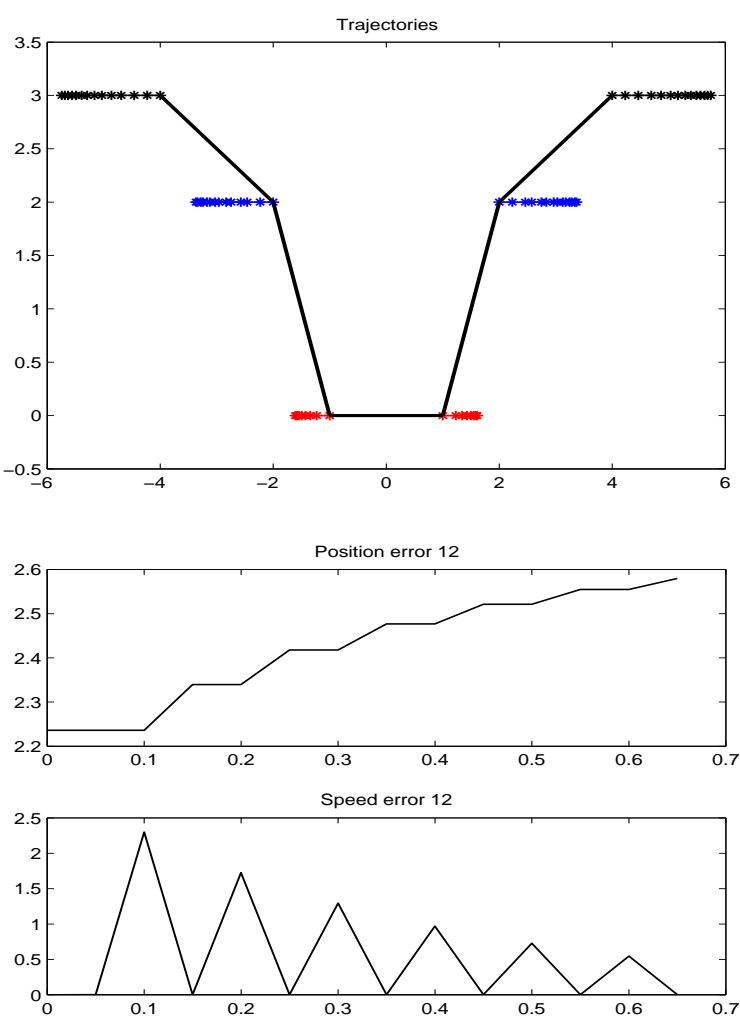

Fig. 3. Flocking: trajectories and errors of the 12 system.
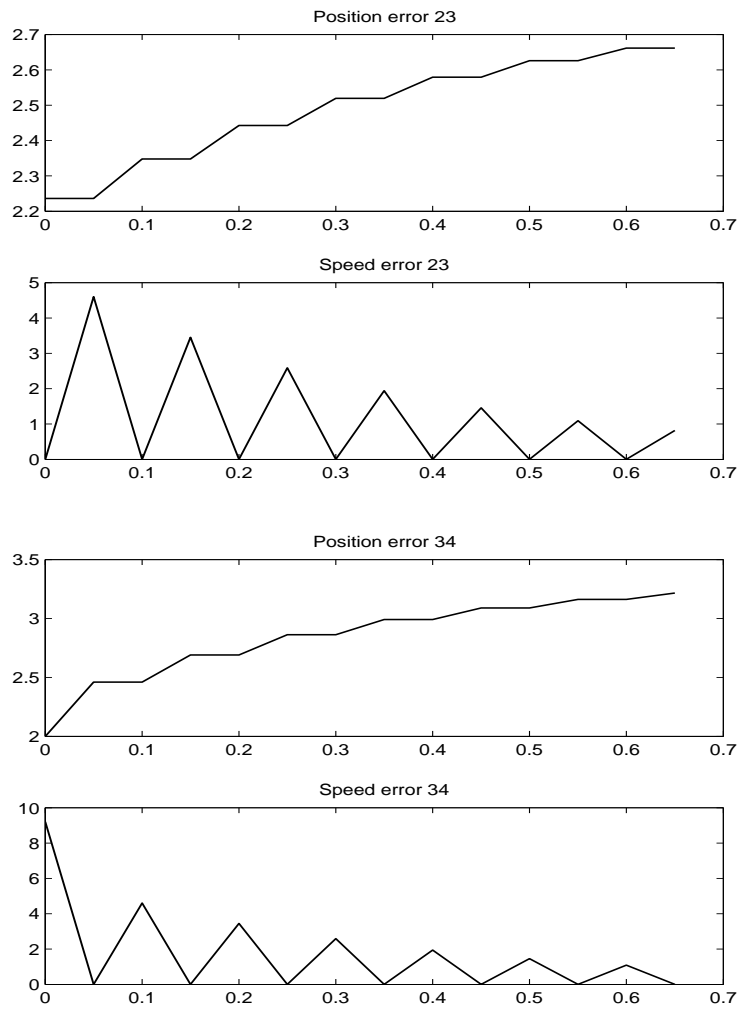

Fig. 4. Flocking: errors of the 23 and the 34 systems. 


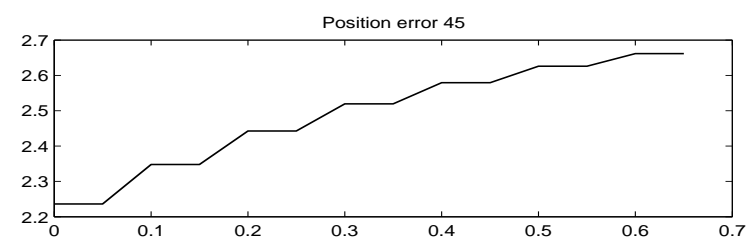

Speed error 45
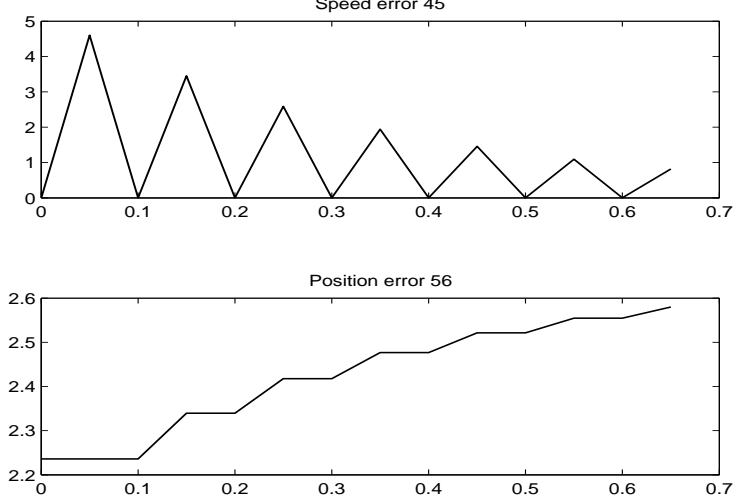

Speed error 56

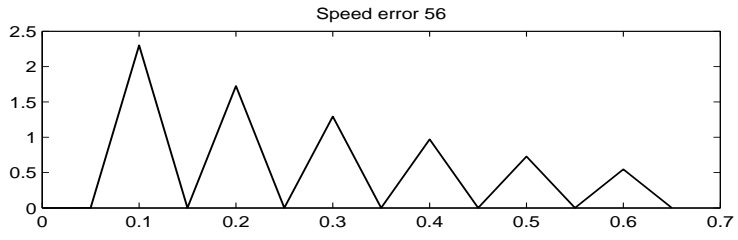

Fig. 5. Flocking: errors of the 45 and the 56 systems.

The performances can be improved by heuristics. For instance, requiring to maintain an euclidean distance inferior to 3.1 even though the connection bound is 3.2. The flocking is reached for $v_{0}=20 v_{c}$, see Figure 6. It is interesting to note that the control action is not able to maintain the error 34 inferior to 3.1 but once the constraint is violated (since the agents are still connected $r=3.2$ ) the priority is to minimize the euclidean distance in order to respect the constraint. Notice how the regularity of the behaviour is lost after the constraint violation, at time 0.55 .

\section{B. Full state consensus}

The control problem formulated in Section IV-B.1 with $q_{x}=10, q_{v}=1$ has admissible solutions for $v_{0}=23 v_{c}$ as shown in Figure 7.

\section{Predictive control strategies}

The control problem formulated in Section IV-B.3 with $N_{p}=3$ works for $v_{0}=21 v_{c}$ but the trajectories are far from ideal. The behaviour is largely improved with $N_{p}=21$, see Figure 8 representing the trajectories and the time evolution of the 34 dynamics for $v_{0}=28 v_{c}$. Notice how the position error of the critical system, the 34 , approaches the bound avoiding the constraint violation, also for an initial speed much higher than those used for the other approaches, i.e. $v_{0}=28 v_{c}$. Furthermore, the evolutions and trajectories present a much smoother and regular behaviour. All these desirable properties are due to the predictive capability of
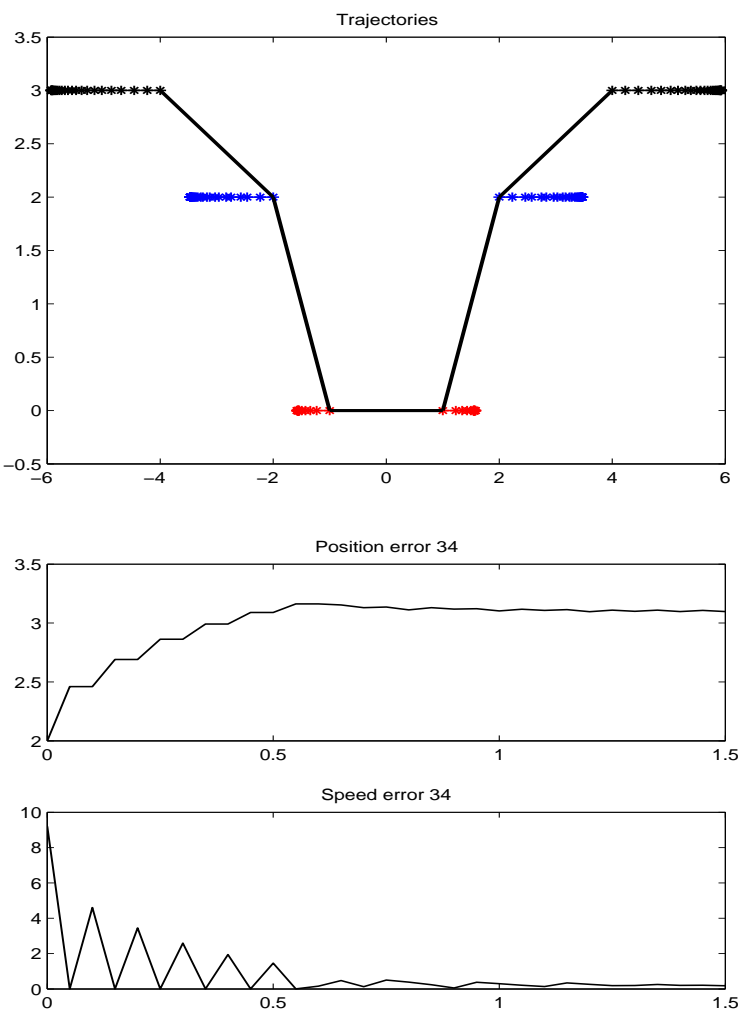

Fig. 6. Flocking: trajectories and errors of the 34 system.
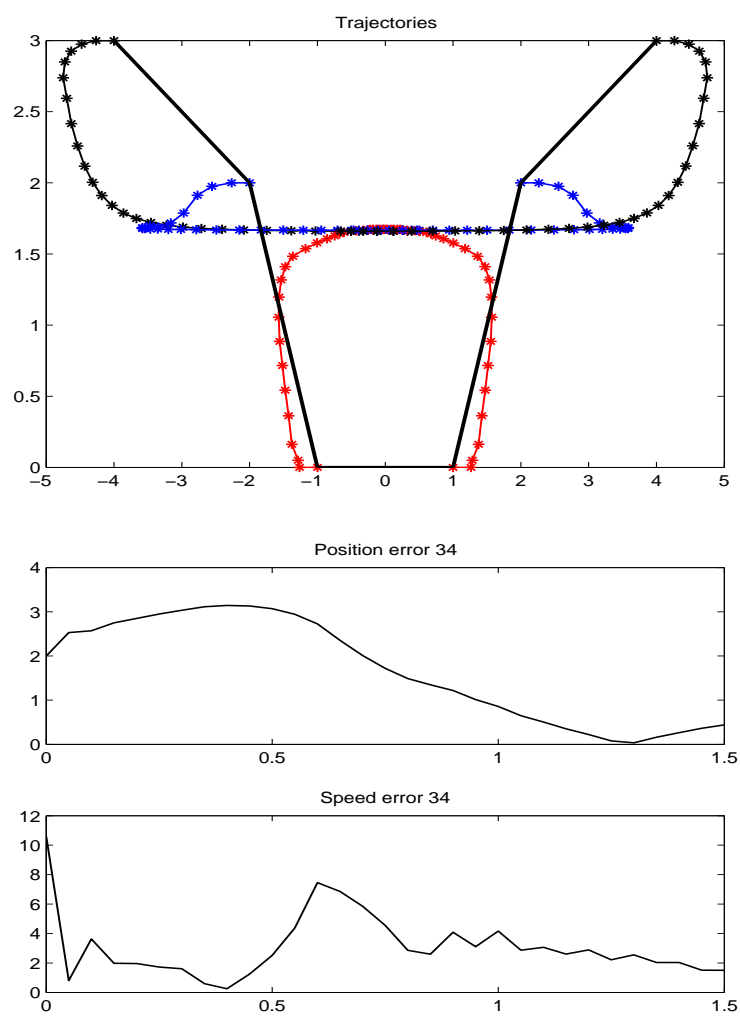

Fig. 7. Consensus: trajectories and errors of the 34 system. 
the approach which permits the control to react to possible violations and to prevent undesired situations in advance.
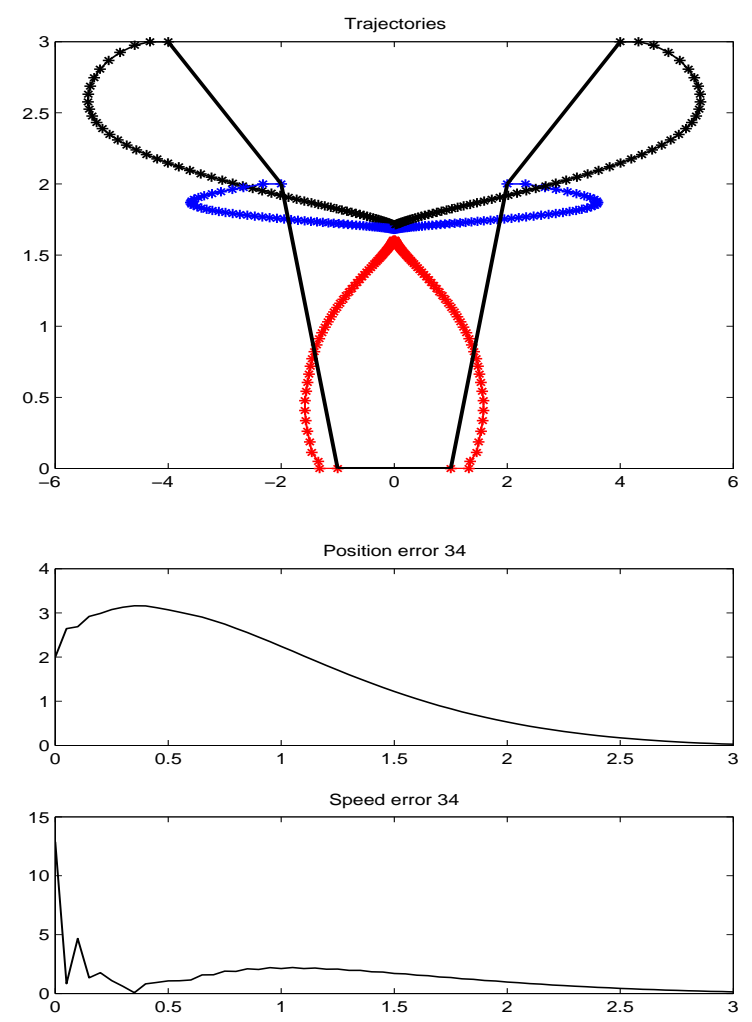

Fig. 8. Predictive control: trajectories and errors of the 34 system.

\section{CONCLUSION AND FURTHER WORKS}

In this paper we presented the results obtained by applying LMI-based conditions for topology preservation to a multiagent system. In particular, a common multi-agent framework has been considered, namely a system composed by several moving agents with limited communication capability. The LMI-conditions demonstrated to allow the connectivity preservation, crucial issue in cooperative control. At the same time, different convex optimization problems have been posed in order to pursue several classical objectives in the multi-agent context, as consensus, flocking and predictive control. The results obtained are proved to improve consistently those achieved with analogous techniques.

Note that the main applications provided in the paper concern fleets of autonomous vehicles. Thus, the size of this associated network does not represent and obstacle for the numerical treatments by LMIs. Moreover, we can choose the network to be preserved as a very sparse one. Consequently, the number of low order LMIs to be solved is of the same order as the network size.

As possible further developments we are considering the design of other control strategies based on the topology preservation conditions, in particular the closed-loop predictive control, and their application to real-time multi-agent systems. Also the extension of the results to the case of nonlinear systems deserves to be considered.

\section{REFERENCES}

[Reynolds(2001)] C. Reynolds. Boids (flocks, herds, and schools: a distributed behavioral model). Website: http://www.red3d.com/cwr/boids/, 2001.

[Blondel et al(2008)] V. D. Blondel, J.-L. Guillaume, R. Lambiotte, and E. Lefebvre. Fast unfolding of communites in large networks. Journal of Statistical Mechanics: Theory and Experiment, P10008(10), 2008.

[Colizza(2007)] V. Colizza, R. Pastor-Satorras, and A. Vespignani. Reaction-diffusion processes and metapopulation models in heterogeneous networks. Nature Physics, 3:276-282, 2007.

[Ratmann et al(2009)] O. Ratmann, C. Wiuf, and J. W. Pinney. From evidence to inference: Probing the evolution of protein interaction networks. HFSP Journal, 3(5):290-306, 2009.

[Pavlopoulos et al(2011)] G.A. Pavlopoulos, M. Secrier, C.N. Moschopoulos, T.G. Soldatos, S. Kossida, J. Aerts, R. Schneider, and P. G. Bagos. Using graph theory to analyze biological networks. BioData Mining, 4(10):1-27, 2011.

[Pecora \& Carroll(1998)] L. M. Pecora and T. L. Carroll. Master stability functions for synchronized coupled systems. Phys. Rev. Lett., 80(10):2109-2112, 1998 .

[Jadbabaie et al(2003)] A. Jadbabaie, J. Lin, and A. S. Morse. Coordination of groups of mobile autonomous agents using nearest neighbor rules. IEEE Trans. on Automatic Control, 48(6):988-1001, 2003.

[Olfati-Saber \& Murray(2004)] R. Olfati-Saber and R.M. Murray. Consensus problems in networks of agents with switching topology and time-delays. IEEE Transactions on Automatic Control, 49:1520-1533, 2004.

[Ren et al(2005)] W. Ren, R. W. Beard, and E. M. Atkins. A survey of consensus problems in multi-agent coordination. In Proceedings of the 2005 American Control Conference, volume 3, pages 1859-1864, 2005.

[Moreau(2005)] L. Moreau. Stability of multiagent systems with timedependent communication links. IEEE Trans. on Automatic Control, 50(2):169-182, 2005.

[Olfati-Saber et al(2007] R. Olfati-Saber, J. A. Fax, and R. M. Murray. Consensus and cooperation in networked multi-agent systems. Proceedings of the IEEE, 95(1):215-233, 2007.

[Morărescu et al(2012)] I.-C. Morărescu, S.-I. Niculescu, and A. Girard. Consensus with constrained convergence rate and time-delays., volume 423 of Lecture Notes in Control and Information Sciences, pages 417-428. Springer Berlin Heidelberg, 2012.

[Shakkottai \& Srikant(2007)] S. Shakkottai and R. Srikant. Network Optimization and Control. Foundations and Trends in Networking. NoW Publishers, 2007.

[Ren \& Beard(2005)] W. Ren and R. W. Beard. Consensus seeking in multiagent systems under dynamically changing interaction topologies. IEEE Trans. on Automatic Control, 50(5):655-661, 2005.

[Morărescu \& Girard(2011)] I.-C. Morărescu and A. Girard. Opinion dynamics with decaying confidence: Application to community detection in graphs. IEEE Trans. on Automatic Control, 56(8):1862 - 1873, 2011.

[Touri and Nedic(2012)] B. Touri and A. Nedic. On approximations and ergodicity classes in random chains. IEEE Transactions on Automatic Control, 57(11):2718-2730, 2012.

[Zavlanos \& Pappas(2008)] M.M. Zavlanos and G.J. Pappas. Distributed connectivity control of mobile networks. IEEE Transactions on Robotics, 24(6):1416-1428, 2008.

[Bullo et al(2009)] F. Bullo, J. Cortés, and S. Martinez. Distributed Control of Robotic Networks. A Mathematical Approach to Motion Coordination Algorithms. Princeton University Press, 2009.

[Fiacchini \& Morărescu(2012)] M. Fiacchini and I.-C. Morărescu. Set theory based condition in LMI form for network topology preservation for decentralized control. In Proc. of the 51st IEEE Conference on Decision and Control (CDC), pages 6229-6234, 2012.

[Fiacchini \& Morărescu(2014)] M. Fiacchini and I.-C. Morărescu. Convex conditions on decentralized control for graph topology preservation. IEEE Transactions on Automatic Control, DOI10.1109/TAC.2013.2292719.

[Fiacchini et al(2010)] M. Fiacchini, T. Alamo, and E. F. Camacho. On the computation of convex robust control invariant sets for nonlinear systems. Automatica, 46(8):1334-1338, 2010.

[Fiacchini et al(2011)] M. Fiacchini, S. Tarbouriech, and C. Prieur. Polytopic control invariant sets for continuous-time systems: A viability theory approach. In Proceedings of the American Control Conference, 2011. ACC'11, pages 1218-1223, San Francisco, CA, june 2011. 
[Boyd et al(1994)] S. Boyd, L. El Ghaoui, E. Feron, and V. Balakrishnan. Linear Matrix Inequalities in system and control theory. SIAM, 1994.

[Boyd \& Vandenberghe(2004)] S. Boyd and L. Vandenberghe. Convex Optimization. Cambridge University Press, 2004.

[Martin \& Girard(2010)] S. Martin and A. Girard In Proceedings of the 49th IEEE Conference on Decision and Control, pages 6293-6298, Atlanta, GA, Dec. 2010. 